\title{
Calcium release channel RyR2 regulates insulin release and glucose homeostasis
}

\author{
Gaetano Santulli, ${ }^{1}$ Gennaro Pagano, ${ }^{2,3,4}$ Celestino Sardu, ${ }^{5,6,7}$ Wenjun Xie, ${ }^{1}$ Steven Reiken, ${ }^{1}$ Salvatore Luca D'Ascia, ${ }^{8}$ \\ Michele Cannone, ${ }^{9}$ Nicola Marziliano, ${ }^{10,11}$ Bruno Trimarco, ${ }^{12}$ Theresa A. Guise, ${ }^{13}$ Alain Lacampagne, ${ }^{14}$ and Andrew R. Marks ${ }^{1,15}$ \\ 'Department of Physiology and Cellular Biophysics, Clyde and Helen Wu Center for Molecular Cardiology, College of Physicians and Surgeons, Columbia University Medical Center, New York, New York, USA. \\ 2Department of Medicine, Imperial College, London, United Kingdom. ${ }^{3}$ Department of Medicine and Health Sciences, University of Molise, Campobasso, Italy. ${ }^{4}$ Department of Translational Medical Sciences, \\ "Federico II" University, Naples, Italy. 5Department of Electrophysiology, the Leiden University Medical Center (LUMC), Leiden, Netherlands. Department of Medical, Surgical, Neurological, Metabolic, \\ and Geriatric Sciences, Second University of Naples, Naples, Italy. 'Department of Cardiovascular Disorders, Catholic University of the Sacred Heart, "John Paul II" Foundation for Research and Treatment, \\ Campobasso, Italy. ${ }^{8}$ Department of Cardiology and Arrhythmology, Clinical Institute "Città Studi" Hospital, Milan, Italy. ${ }^{9}$ Division of Cardiology, G. Tatarella Hospital, Cerignola (Foggia), Italy. ${ }^{10}$ Division of \\ Molecular Pathology, Niguarda Ca' Granda Hospital, Milan, Italy. "Divisions of Medical Genetics and Cardiology, University Hospital of Parma, Parma, Italy. ${ }^{12 D e p a r t m e n t ~ o f ~ A d v a n c e d ~ B i o m e d i c a l ~ S c i e n c e s, ~}$ \\ "Federico II" University, Naples, Italy. ${ }^{13}$ Department of Medicine, Division of Endocrinology, Indiana University School of Medicine, Indianapolis, Indiana, USA. ${ }^{14}$ U1046 INSERM, UMR 9214, CNRS, \\ CHRU Montpellier, Montpellier, France. ${ }^{15}$ Department of Medicine, Columbia University, New York, New York, USA.
}

\begin{abstract}
The type 2 ryanodine receptor (RyR2) is a $\mathrm{Ca}^{2+}$ release channel on the endoplasmic reticulum (ER) of several types of cells, including cardiomyocytes and pancreatic $\beta$ cells. In cardiomyocytes, RyR2-dependent $\mathrm{Ca}^{2+}$ release is critical for excitation-contraction coupling; however, a functional role for RyR2 in $\beta$ cell insulin secretion and diabetes mellitus remains controversial. Here, we took advantage of rare RyR2 mutations that were identified in patients with a genetic form of exercise-induced sudden death (catecholaminergic polymorphic ventricular tachycardia [CPVT]). As these mutations result in a "leaky" RyR2 channel, we exploited them to assess RyR2 channel function in $\beta$ cell dynamics. We discovered that CPVT patients with mutant leaky RyR2 present with glucose intolerance, which was heretofore unappreciated. In mice, transgenic expression of CPVT-associated RyR2 resulted in impaired glucose homeostasis, and an in-depth evaluation of pancreatic islets and $\beta$ cells from these animals revealed intracellular $\mathrm{Ca}^{2+}$ leak via oxidized and nitrosylated RyR2 channels, activated ER stress response, mitochondrial dysfunction, and decreased fuel-stimulated insulin release. Additionally, we verified the effects of the pharmacological inhibition of intracellular Ca ${ }^{2+}$ leak in CPVT-associated RyR2-expressing mice, in human islets from diabetic patients, and in an established murine model of type 2 diabetes mellitus. Taken together, our data indicate that RyR2 channels play a crucial role in the regulation of insulin secretion and glucose homeostasis.
\end{abstract}

\section{Introduction}

The molecular mechanisms underlying insulin secretion and glucose metabolism have not been fully elucidated (1-6). Numerous studies have demonstrated that calcium $\left(\mathrm{Ca}^{2+}\right)$ plays a pivotal role in insulin secretion from the islets of Langerhans and that altered cellular $\mathrm{Ca}^{2+}$ homeostasis may be involved in defective insulin release (7-11). Nevertheless, according to the classical view of the glucose-sensing machinery, insulin secretion largely depends on voltage-activated $\mathrm{Ca}^{2+}$ influx, whereas the role of intracellular $\mathrm{Ca}^{2+}$ release in pancreatic $\beta$ cells in response to glucose has not been fully elucidated (12-14). In particular, the mechanistic role of type 2 ryanodine receptor/ $\mathrm{Ca}^{2+}$ release channel (RyR2), which is expressed on the endoplasmic reticulum (ER) of pancreatic $\beta$ cells (14-16), in insulin secretion remains controversial, and a relationship between RyR2 and type 2 diabetes mellitus (T2DM) has not been clearly established.

Conflict of interest: Andrew R. Marks is a consultant and member of the board of ARMGO Pharma Inc., which is targeting RyR channels for therapeutic purposes. Submitted: September 29, 2014; Accepted: February 27, 2015.

Reference information: J Clin Invest. 2015;125(5):1968-1978. doi:10.1172/JCI79273.
RyR channels are macromolecular complexes comprising 4 protomers, each with a molecular mass of $565 \mathrm{kDa}(17)$. They are the largest known ion channels, and the structure of RyR1, which is highly homologous ( $70 \%)$ to RyR2, has recently been solved, revealing that RyRs are members of the 6-transmembrane family of ion channels (18). RyR channels are widely expressed and regulate the release of intracellular $\mathrm{Ca}^{2+}$ involved in vital organspecific functions (17-22). We studied knockin mice harboring RyR2 containing mutations that were discovered in humans with a genetic form of exercise-induced sudden cardiac death syndrome known as catecholaminergic polymorphic ventricular tachycardia (CPVT), which has a $50 \%$ mortality rate by 35 years of age (23-25). Each RyR2 protomer binds one calstabin2, a subunit that stabilizes the closed state of the channel and prevents the pathological leak of $\mathrm{Ca}^{2+}(18,19,26)$. Dissociation of calstabin2 from RyR2 results in intracellular $\mathrm{Ca}^{2+}$ leak via RyR2 channels $(20,23,27)$. We have demonstrated that CPVT-linked RYR2 mutations reduce the binding affinity of the stabilizing subunit calstabin2 to the RyR2 channel $(17,20,23)$.

CPVT patients provide a unique opportunity to address the role of RyR2 in glucose metabolism because they have disease- 
A

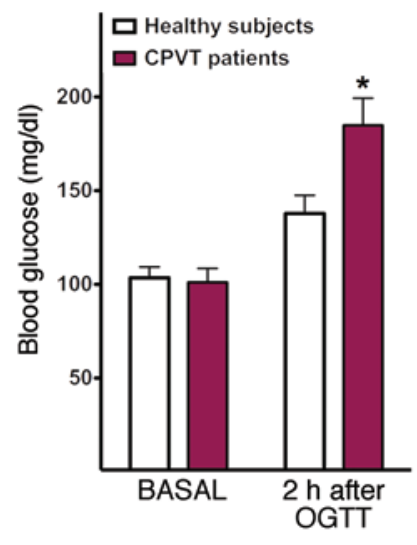

B

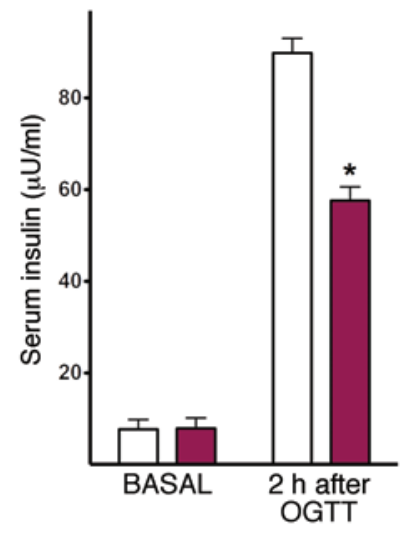

C

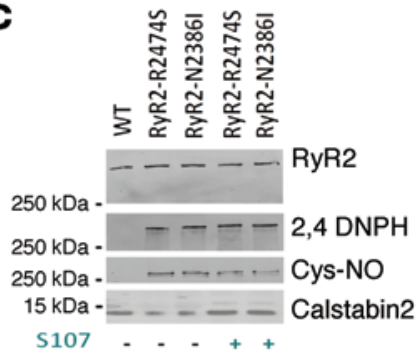

E

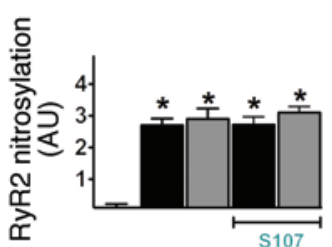

D

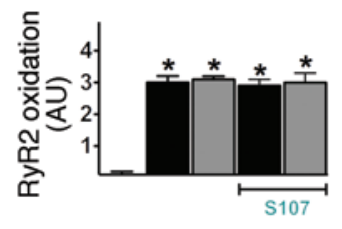

G

\section{ㅁ WT \\ - RyR2-R2474S \\ 口 RyR2-N2386I}

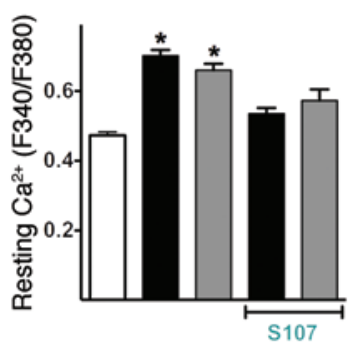

H

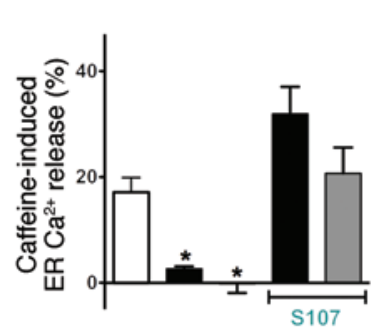

I

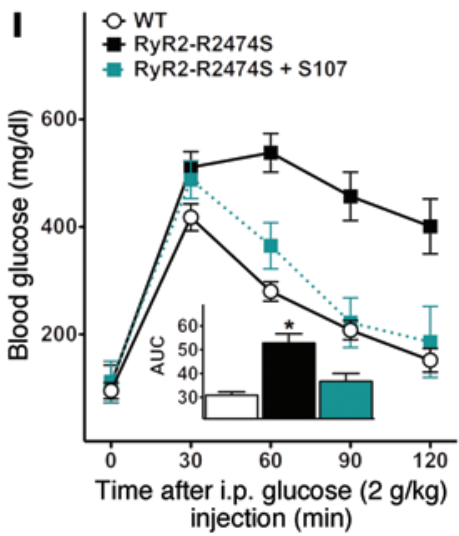

$\mathbf{F}$

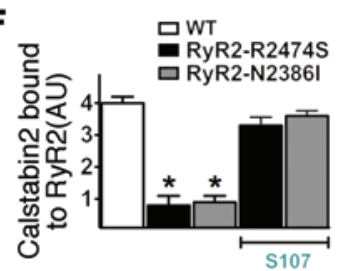

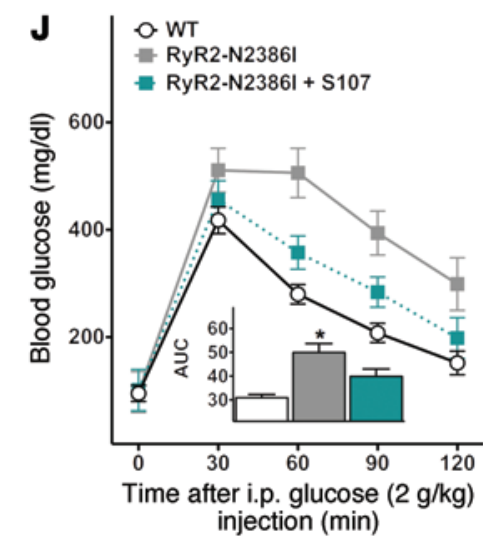
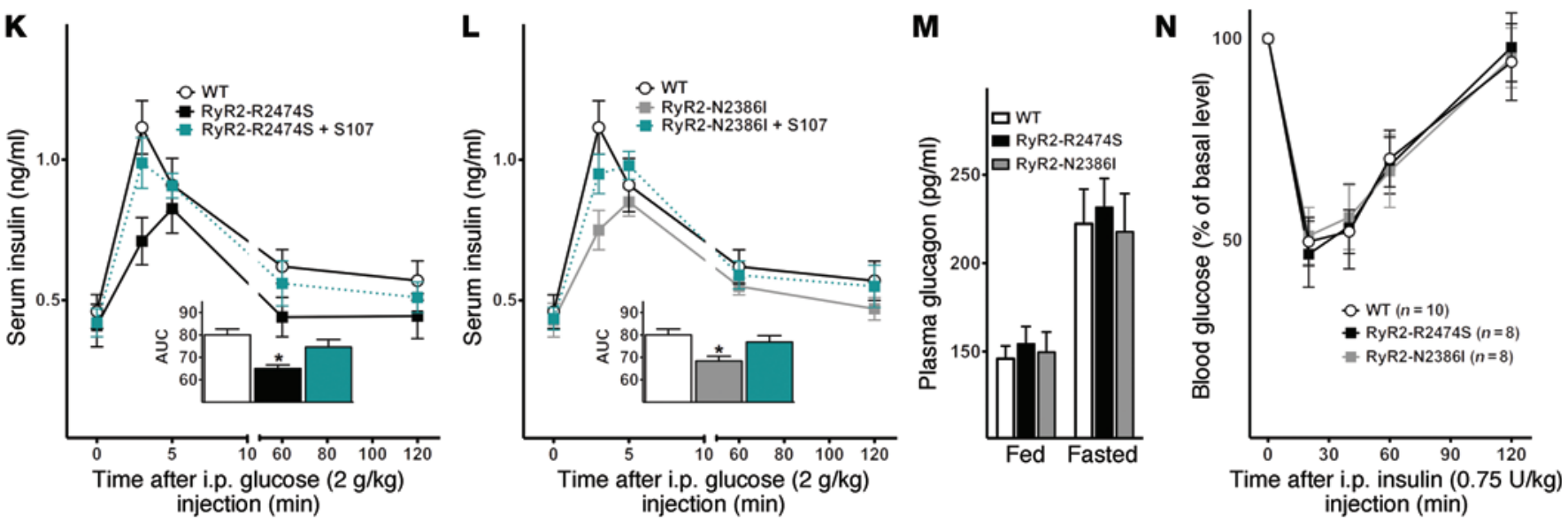

Figure 1. Humans and mice with leaky RyR2 channels exhibit glucose intolerance. OGTT in healthy subjects (see Supplemental Table 1 for patient characteristics) and patients with CPVT due to RYR2 mutations (see Supplemental Table 2 for RYR2 mutations in CPVT patients) with determination of blood glucose (A) and serum insulin levels (B). ${ }^{*} P<0.05$ compared with healthy subjects, 2-tailed Student's $t$ test. (C) Representative immunoblots of pancreatic islets from CPVT mice chronically (50 mg/kg/d, 4 weeks) treated with S107. 2,4 DNPH, 2,4-dinitrophenylhydrazone; Cys-NO, nitrosylation. (D-F) Quantification of data shown in $\mathbf{C}$ (from triplicate experiments). ( $\mathbf{G}$ and $\mathbf{H})$ Resting cytosolic $\left[\mathrm{Ca}^{2+}\right]_{\text {cyt }}$ is elevated in pancreatic $\beta$ cells from CPVT mice (pancreatic $\beta$ cell $\mathrm{Ca}^{2+}$ imaged using acetoxy-methyl-ester fura-2, fura-2 AM). (C) ER Ca ${ }^{2+}$ stores measured using caffeine to release the RyR2 $\mathrm{Ca}^{2+}$ pool are markedly depleted in pancreatic $\beta$ cells from CPVT mice (H); acute (10 $\mu \mathrm{M}, 4$ hours) S107 treatment restores ER Ca ${ }^{2+}$ by inhibiting RyR2-mediated leak. (I and J) Blood glucose levels following i.p. glucose challenge in WT, RyR2-R2474S, and RyR2-N2386I mice chronically treated with S107. Insulin concentration measured in sera from WT, RyR2-R2474S (K), and RyR2-N2386I (L) mice, following i.p. glucose challenge, with and without chronic S107. (M) Plasma glucagon in fed and fasted (6 hours) conditions. (N) Blood glucose levels measured following i.p. insulin injection. Insets in I-L represent AUC. Data are expressed as mean \pm SEM. $n=9-12$ /group. ${ }^{*} P<0.05$ compared with WT, ANOVA, Tukey-Kramer post hoc correction. Additional details are given in Methods. 
causing mutations that make the channels leaky $(23,24)$. Moreover, we have developed a new class of orally available small molecule drugs (Rycals), which are 1,4-benzothiazepine derivatives that inhibit stress-induced (e.g., oxidation, nitrosylation, or hyperphosphorylation of RyR2) or genetic mutation-induced dissociation of calstabin (e.g., CPVT mutations) from RyR channels (17, 27, 28). The Rycal tested in the present study (S107) is in the same chemical class and has the same mechanism of action as 2 closely related Rycals that are undergoing clinical testing for cardiovascular and muscle disorders.

\section{Results}

Patients with leaky RyR2 channels have glucose intolerance and impaired insulin secretion. Oral glucose tolerance tests (OGTT) performed in 27 CPVT patients with known RYR2 mutations and 27 age- and BMI-matched healthy controls (Supplemental Tables 1 and 2; supplemental material available online with this article; doi:10.1172/JCI79273DS1) revealed significant glucose intolerance (Figure 1A) and reduced insulin levels after glucose challenge in CPVT patients (Figure 1B).

To determine whether the mutant leaky RyR2 can cause the glucose intolerance observed in CPVT patients, we studied 2 knockin mouse models, each harboring 1 of the human CPVTlinked RYR2 mutations found in the 27 patients with glucose intolerance, RyR2-R2474S or RyR2-N2386I. RyR2 was present in murine and human pancreatic islets (Supplemental Figure 1, A-C, and Supplemental Tables 3 and 4), consistent with previous analyses (16). We have previously shown that mutant CPVT RyR2 channels are leaky due to depletion of the RyR2-stabilizing subunit calstabin2 (FKBP12.6) from the RyR2 macromolecular complex and that oxidation and/or Cys-nitrosylation of RyR2 also causes depletion of calstabin2 from the channel $(17,20)$. RyR2 channels from murine CPVT islets were oxidized, nitrosylated, and depleted of calstabin2 (Figure 1, C-F). These findings support a previous report suggesting that genetic ablation of calstabin 2 is associated with impaired insulin secretion in pancreatic $\beta$ cells (29). The caffeine-depletion assay, in which caffeine is applied to cells to open the RyR2 channel in order to measure the amount of $\mathrm{Ca}^{2+}$ released from the ER, revealed that $\mathrm{ER} \mathrm{Ca}^{2+}$ stores were dramatically depleted in pancreatic islets from mice with leaky RyR2 (Figure 1, $\mathrm{G}$ and $\mathrm{H}$ ). To directly measure the $\mathrm{Ca}^{2+}$ leak, we used an assay in which RyR2-containing microsomes were isolated from pancreatic islets and placed in a $\mathrm{Ca}^{2+}$-sensitive dye-containing solution. The microsomes were loaded with $\mathrm{Ca}^{2+}$ using ATP to activate the $\mathrm{Ca}^{2+}$ uptake pump, which was then blocked using thapsigargin; the amount of RyR2-specific $\mathrm{Ca}^{2+}$ leak was measured as an increase in fluorescence outside the microsomes (Supplemental Figure 2). Ryanodine was used to block the leak to show that it was due to the RyR channels. Thus, both of the CPVT-linked RyR2 mutations resulted in depleted $\mathrm{ER} \mathrm{Ca}^{2+}$ stores as a consequence of the intracellular $\mathrm{Ca}^{2+}$ leak in pancreatic islets (Figure 1, G and $\mathrm{H}$, and Supplemental Figure 2).

Leaky $R y R 2$ causes impaired insulin secretion in vivo. Similarly to CPVT patients, both CPVT murine models examined here exhibited marked glucose intolerance (Figure 1, I and J) and decreased glucose-stimulated serum insulin levels compared with WT littermates (Figure 1, K and L). There were no significant differences in baseline parameters (Supplemental Table 5). The release of C-peptide in response to glucose was significantly decreased in CPVT mice compared with WT littermates (Supplemental Figure $3 \mathrm{~A})$, whereas plasma glucagon levels (Figure 1M) and gluconeogenic response were comparable among groups (Supplemental Figure 3B). Insulin tolerance tests were normal, indicating that glucose intolerance was not due to insulin resistance (Figure 1N). Besides, islet architecture was similar in WT and CPVT mice (Figure 2A). Pancreas weight, islet size, insulin content, and $\beta$ cell mass in CPVT mice were comparable to those observed in WT littermates (Figure 2, B-G), further denoting that the decrease in serum insulin levels was most likely due to impaired insulin secretion. To better assess this aspect, we also performed ex vivo secretion assays in isolated islets. In the presence of leaky RyR2 channels, insulin release was impaired in response to glucose or leucine and glutamine (Figure 2, G-I) and was rescued following the pharmacological blockage of ATP-sensitive potassium $\left(\mathrm{K}_{\mathrm{ATP}}\right)$ channels with glyburide (glibenclamide, Figure 2J). Importantly, glucagon content and secretion were similar among the studied groups (Supplemental Figure 4, A and B).

Leaky RyR2 channels cause ER stress and defective mitochondrial function, impairing metabolism-secretion coupling. Given the relationship between intracellular $\mathrm{Ca}^{2+}$ storage and ER stress response $(30,31)$, we investigated the activation of the unfolded protein stress response in our CPVT models. Interestingly, we found that intracellular $\mathrm{Ca}^{2+}$ leak via RyR2 and depleted ER stores were associated with a significant increase in immunoglobin heavy-chain binding protein (BiP, a chemical chaperone) and in total and spliced X-box binding protein 1 (XBP1), but not in CCAAT/enhancer-binding protein homologous protein (CHOP), which is considered an execution signal that triggers apoptosis (Supplemental Figure 5, A-D). Importantly, no significant changes were observed in the levels of other key modulators of $\mathrm{Ca}^{2+}$ handling (Supplemental Figure 6). These data suggest a mild adaptive response that favors a survival outcome as opposed to the terminal response in which upregulation of CHOP leads to apoptosis. The activation of the ER stress response along with the impaired insulin secretion observed in CPVT islets in response to the fuel secretagogues leucine and glutamine (Figure 2I), which stimulate insulin release through increased mitochondrial metabolism and ATP production $(4,32)$, prompted us to investigate the mitochondria in pancreatic $\beta$ cells. Mitochondrial morphology was abnormal in CPVT compared with WT $\beta$ cells (fragmented cristae, swelling, lamellar degeneration, and outer membrane disruption) (Figure 3A). There were no significant differences in the diameter or number of insulin secretory granules (Supplemental Figure 7, A and B). However, total mitochondrial area was increased (Figure 3B), cristae density reduced (Figure $3 \mathrm{C}$ ), the percentage of abnormal mitochondria augmented (Figure 3D), mitochondrial DNA (mtDNA) copy number reduced (Figure 3E), and ROS generation increased (Figure 3F) in CPVT versus WT $\beta$ cells. Further evaluation of specific markers of mitochondrial dysfunction revealed an increased expression of uncoupling protein 2 (Ucp2) (Figure 3G) in CPVT islets, which negatively regulates insulin release and is considered an essential link between $\beta$ cell dysfunction, T2DM, and obesity (33-35), and reduced aconitase activity (Figure $3 \mathrm{H}$ ) accompanied by decreased mRNA levels of aconitase2 (Supplemental Figure 8A), enhanced 
A
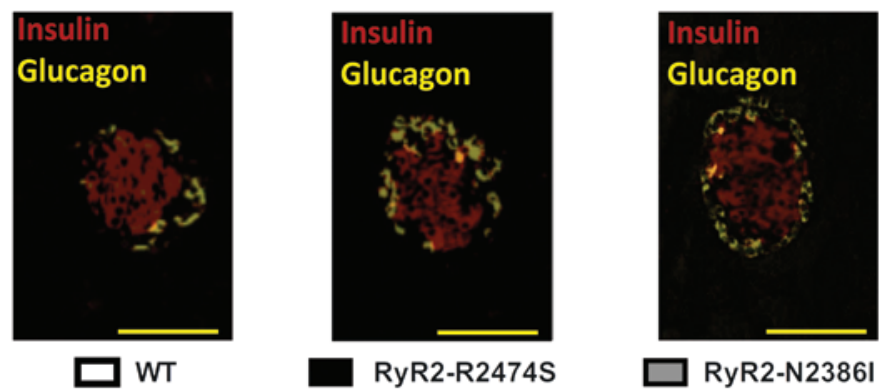

B

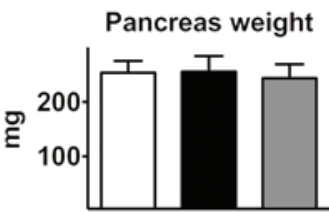

C
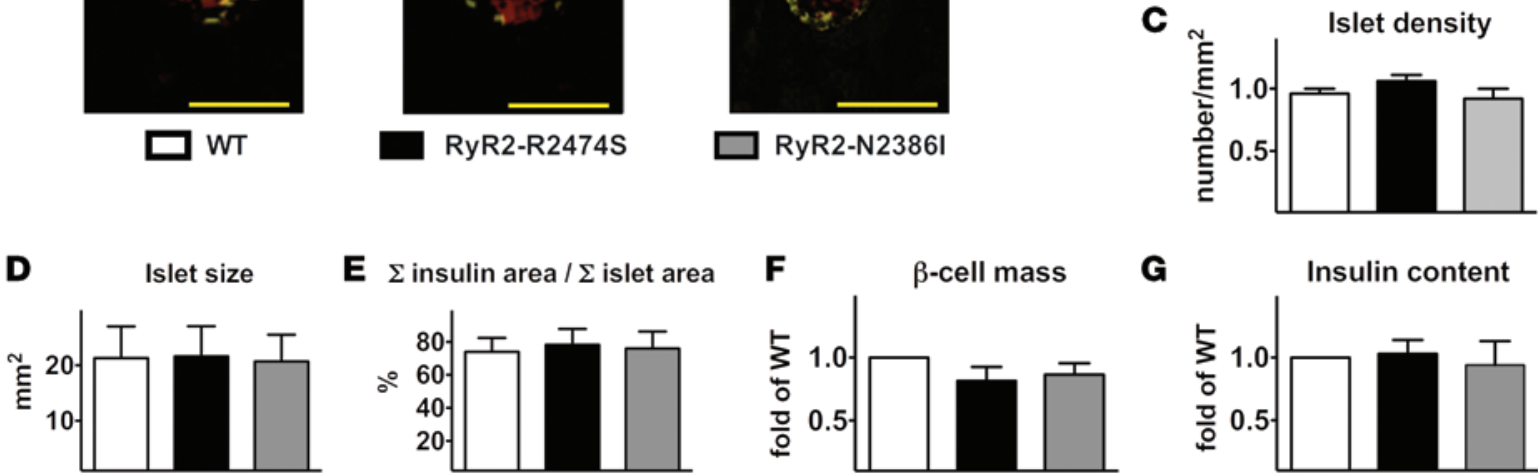

E $\Sigma$ insulin area $/ \Sigma$ islet area

$\mathbf{F}$

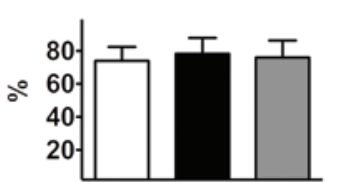

H

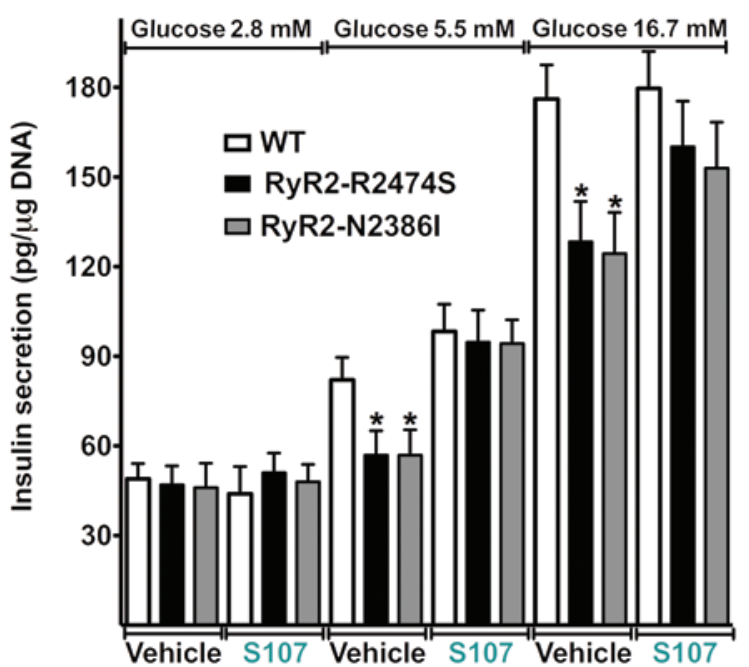

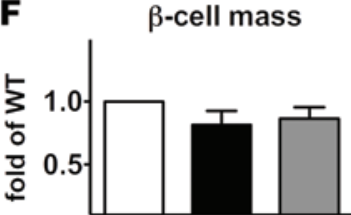

G

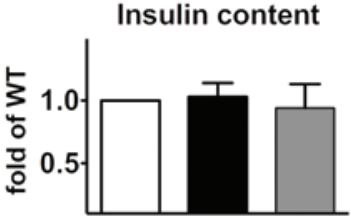

I

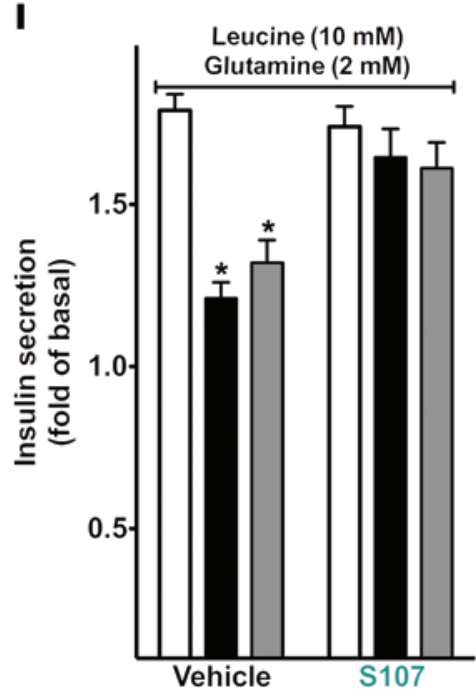

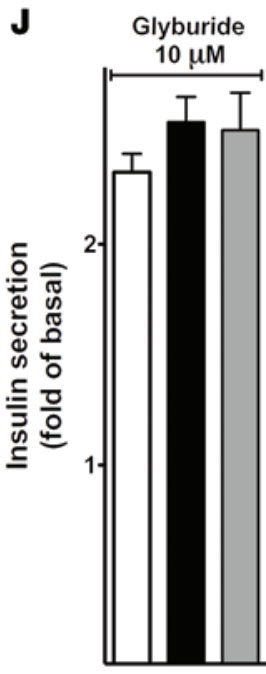

Figure 2. Leaky RyR2 channels cause impaired insulin secretion. (A) Representative images of pancreatic islets from WT, RyR2-R2474S, and RyR2N2386I mice stained for insulin (red) and glucagon (yellow). Original magnification, $\times 63$. Scale bars: $200 \mu$ m. Pancreatic weight (B), islet density (C) and size (D), ratio of $\Sigma$ insulin area/ $\Sigma$ islet area $(\mathbf{E}), \beta$ cell mass $(\mathbf{F})$, and insulin content (G). Data are expressed as mean \pm SEM. Additional details are given in Methods. (G-J) Insulin release in response to glucose (H), leucine plus glutamine (I), or glyburide (J) evaluated ex vivo in pancreatic islets isolated from WT and CPVT mice undergoing $S 107$ ( $50 \mathrm{mg} / \mathrm{kg} / \mathrm{d}, 4$ weeks) or vehicle treatment. Data are expressed as mean $\pm \mathrm{SEM} . n=6-10$ animals $/$ group. ${ }^{*} P<0.05 \mathrm{vs}$. WT, ANOVA, Tukey-Kramer post hoc correction.

expression of peroxisome proliferator-activated receptor gamma coactivator-1 $\alpha$ (Pgc1a) (Supplemental Figure 8B), and reduced levels of mitochondrial $\mathrm{Ca}^{2+}$ uniporter $(\mathrm{Mcu})$ (Supplemental Figure 8C), mitochondrial pyruvate carriers 1 and 2 (Mpc1-2) (Supplemental Figure 8D), and Atp6 (Figure 3I), a subunit of the mitochondrial pump ATP synthase. These findings were mirrored by decreased glucose-induced (Figure 3J) and pyruvate-induced (Figure 3K) ATP production, strongly suggesting a respiratory chain deficiency. Decreased ATP production prompted us to evaluate the regulation of $\mathrm{K}_{\mathrm{ATP}}$ channels, fundamental regulators of insulin release (5, 36). CPVT islets exhibited increased Kir6.2 mRNA and protein, which encodes a component of the $\mathrm{K}_{\text {ATP }}$ channel critical to nutrientsecretion coupling, whereas levels of the regulatory subunit SUR1 were not significantly altered (Supplemental Figure 9). As an addi- tional indicator of mitochondrial function, we monitored NADPH autofluorescence, increase of which in response to glucose was significantly impaired in both RyR2-R2474S and RyR2-N2386I mutants, indicating a relative energy deficit (Supplemental Figure

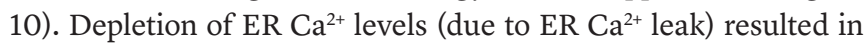
reduced glucose-stimulated mitochondrial $\mathrm{Ca}^{2+}$ uptake in pancreatic $\beta$ cells from CPVT mice (Figure 4, A-C). Similarly, mitochondrial $\mathrm{Ca}^{2+}$ uptake was also reduced following application of caffeine, which releases the $\mathrm{ER} \mathrm{Ca}^{2+}$ stores by activating RyR2 (Figure $4, \mathrm{D}-\mathrm{F})$. Thus, depletion of $\mathrm{ER} \mathrm{Ca}^{2+}$ stores due to leaky RyR2 channels reduces glucose-induced mitochondrial $\mathrm{Ca}^{2+}$ uptake.

Pharmacologic stabilization of RyR2 improves insulin secretion and glucose tolerance. In order to confirm that intracellular $\mathrm{Ca}^{2+}$ leak via RyR2 leads to decreased insulin secretion, we used 
A
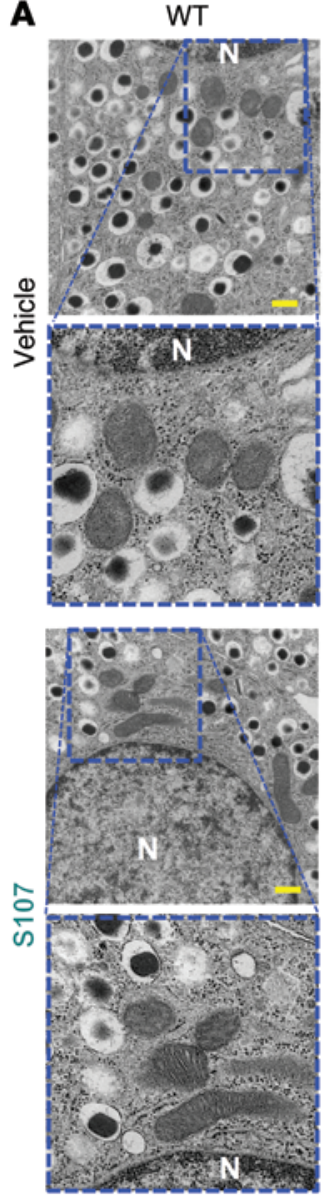

$\mathbf{F}$

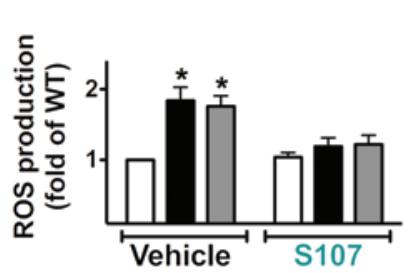

RyR2-R274S
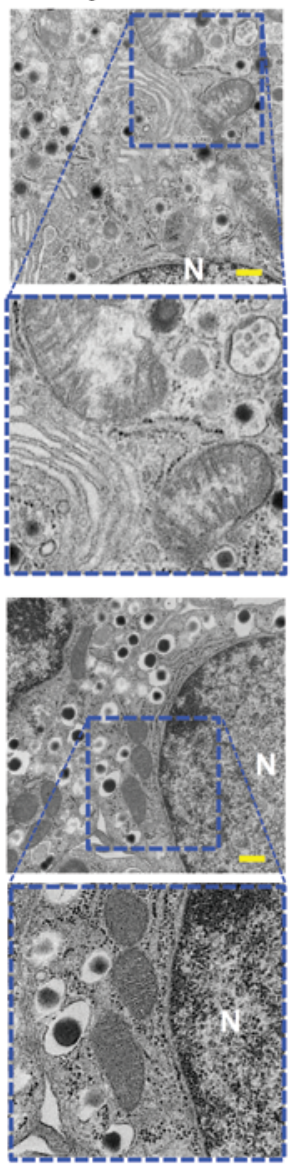

RyR2-N2386I
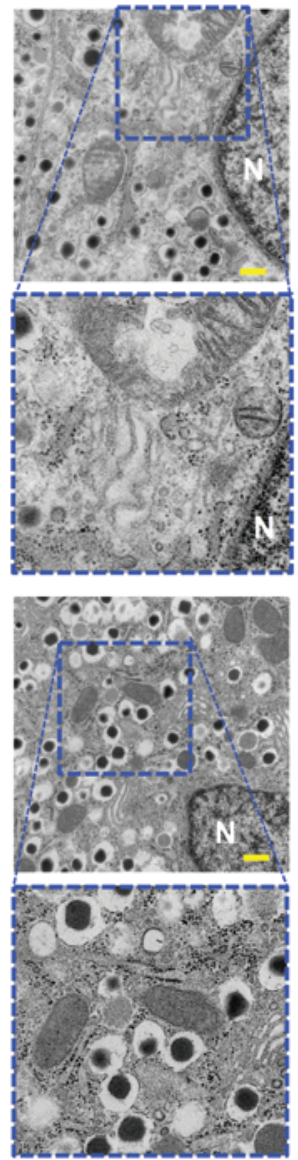

B

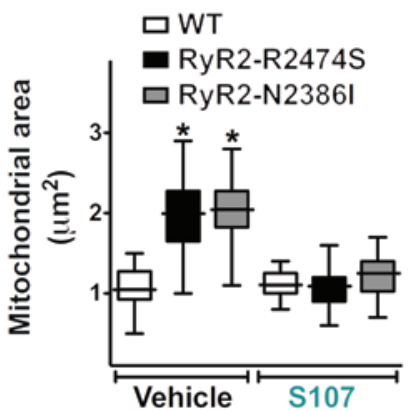

C

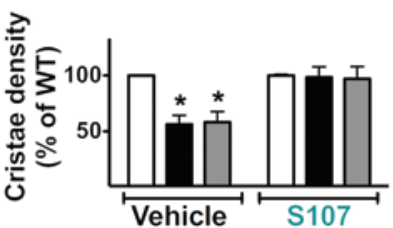

D

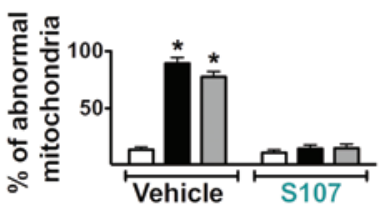

E

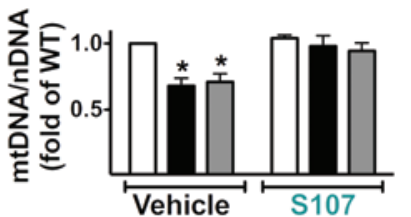

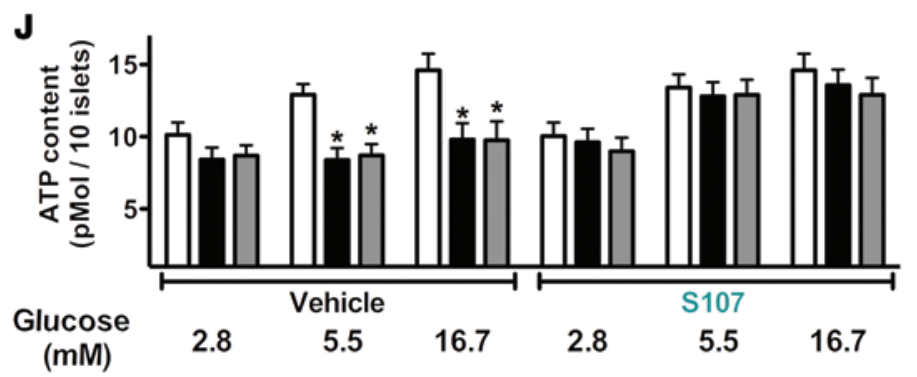

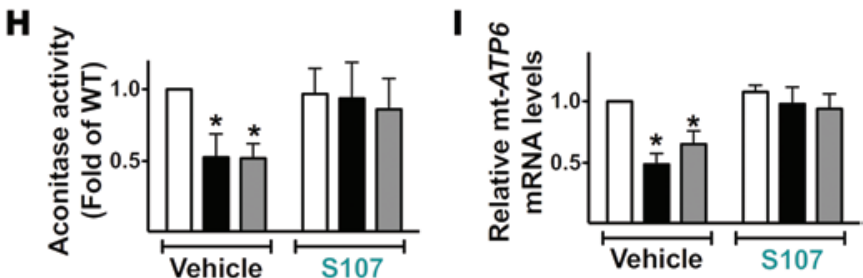

G

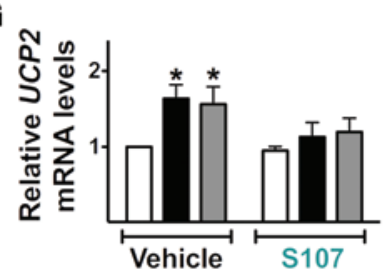

K

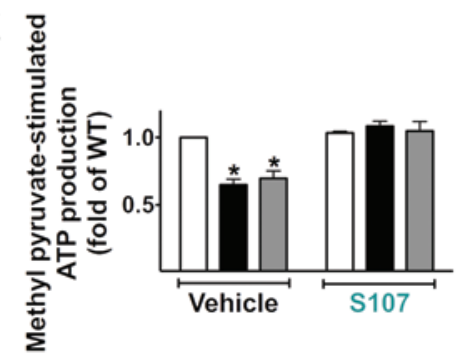

Figure 3. Leaky RyR2 channels lead to abnormal mitochondrial structure and function in pancreatic $\boldsymbol{\beta}$ cells. (A) Representative transmission electron micrographs of pancreatic $\beta$ cell mitochondria from 4-month-old WT, RyR2-R2474S, and RyR2-N2386I mice, treated for 4 weeks with S107 or vehicle. Original magnification, $\times 30,000$; insets, $\times 65000$. N, nucleus. Scale bars: $500 \mathrm{~nm}$. (B-D) Morphometric analyses of mitochondria reveal ultrastructural abnormalities in CPVT mice. (B) Mitochondrial area, (C) cristae density, (D) percentage of abnormal mitochondria per cell (mitochondria were defined as abnormal when a loss of electron density was detectable in more than $20 \%$ of the area of a mitochondrion). (E) mtDNA/nDNA copy number, (F) ROS production, (C) UCP2 expression, (H) aconitase activity, and (I) mt-ATP6 expression in isolated pancreatic islets. (J and K) ATP production in isolated pancreatic islets challenged with glucose (at indicated concentrations, J) or pyruvate $(10 \mathrm{mM}, \mathbf{K})$. Islets were isolated from $n=7-10$ mice per group. Data are shown as mean \pm SEM (triplicate measurements per sample). Box plots in B indicate upper/lower quartiles, lines in the middle of each box are the medians, and the whiskers represent the range of minimum and maximum values of total mitochondrial area per section. ${ }^{*} P<0.05 \mathrm{vs}$. WT, ANOVA, Tukey-Kramer post hoc correction. 

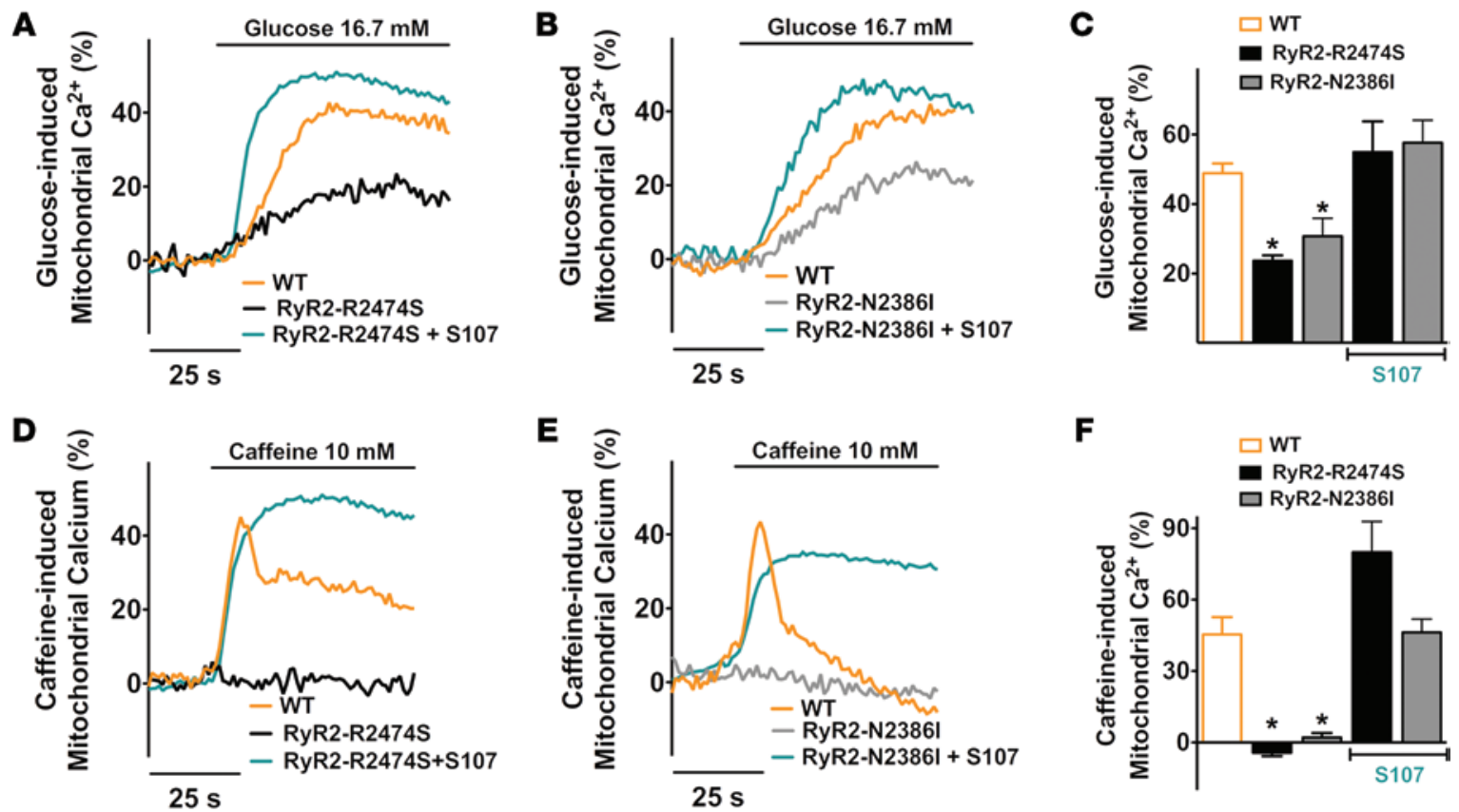

Figure 4. Intracellular $\mathrm{Ca}^{2+}$ leak via RyR2 causes decreased glucose-stimulated mitochondrial $\mathrm{Ca}^{2+}$ uptake, which is restored by Rycal treatment. (A-C) Mitochondrial $\mathrm{Ca}^{2+}$ uptake in response to glucose was decreased in pancreatic $\beta$ cells from 2 CPVT mouse models with leaky RyR2 (representative traces for RyR2-R2474S and RyR2-N2386I are shown in A and B, respectively). S107 treatment (50 mg/kg/d, 4 weeks) improved mitochondrial Ca ${ }^{2+}$ uptake. (D-F) Caffeine-induced mitochondrial $\mathrm{Ca}^{2+}$ uptake was also markedly reduced, reflecting depleted $\mathrm{ER} \mathrm{Ca}^{2+}$ stores, and was improved with $\mathrm{S} 107$ treatment (representative traces for RyR2-R2474S and RyR2-N2386I are shown in D and E, respectively). $n=7-10$ mice per group. Data are shown as mean \pm SEM. ${ }^{*} P<0.05$ vs. WT.

the Rycal S107, which prevents stress-induced dissociation of the stabilizing subunit calstabin2 from RyR2, thereby preventing $\mathrm{ER} \mathrm{Ca}^{2+}$ leak $(27,37)$. S107 is water soluble and has no significant activity against other ion channels, including human ether-a-gogo-related gene (HERG) and voltage-gated $\mathrm{Ca}^{2+}$ channels (28). $\mathrm{S} 107(50 \mathrm{mg} / \mathrm{kg} / \mathrm{d})$ prevented calstabin2 depletion from the RyR2 complex of pancreatic islets (Figure 1, C-E), reduced $\mathrm{ER} \mathrm{Ca}^{2+}$ leak (Figure 1, G and H), improved glucose tolerance (Figure 1, I and $\mathrm{J}$ ), and increased insulin secretion in CPVT mice (Figure 1, K and L). Furthermore, islets isolated from CPVT mice treated with $\mathrm{S107}$ exhibited a secretory response to glucose (Figure $2 \mathrm{H}$ ) and leucine/ glutamine (Figure 2I) that was comparable to that seen in WT. Mitochondrial ultrastructural abnormalities in CPVT islets were also improved by $\mathrm{S107}$ treatment, as were the mtDNA copy number and ROS generation (Figure 3).

Next, we studied the effects of S107 on the RyR2 macromolecular complex in human islets obtained from T2DM and control (nondiabetic) human subjects (Supplemental Table 4). Islets from diabetic patients exhibited oxidation and nitrosylation of RyR2 and depletion of calstabin2 from the channel complex (Figure 5, A-D), similar to that observed in murine CPVT islets (Figure 1, C-F). S107 treatment prevented the dissociation of calstabin2 from RyR2 channels (Figure 5, A and D), but did not affect the oxidation and nitrosylation of RyR2. We then tested S107 in an animal model of T2DM, the $o b / o b$ mouse. Islets of Langerhans isolated from $o b / o b$ mice displayed marked posttranslational modifications of RyR2 (Figure 5, E-H), comparable to that seen in human diabetic (Figure 5, A-C) and CPVT islets (Figure 1, C-F), accompanied by intracellular $\mathrm{Ca}^{2+}$ leak (Supple- mental Figure 11), activation of ER stress (Figure 5I), and overall mitochondrial dysfunction evaluated in terms of Atp6, aconitase2, Ucp2, Pgc1a, Mcu, and Mpc1-2 expression (Supplemental Figure 12, A-F) and ATP synthesis (Supplemental Figure 13). A 4-week treatment with oral $\$ 107$ prevented the loss of calstabin2 from the complex (Figure 5H), improved the $\mathrm{Ca}^{2+}$ leak via RyR2 (Supplemental Figure 11), ER stress response (Figure 5I), mitochondrial dysfunction (Supplemental Figure 12, A-F, and Supplemental Figure 13), and in vivo glucose tolerance (Figure 5J), and also increased glucose-stimulated insulin secretion assessed ex vivo (Figure 5K).

\section{Discussion}

In the present study, we examined glucose homeostasis in 27 CPVT patients, all of whom had RYR2 mutations, including the RyR2R2474S or RyR2-N2386I mutations that were engineered in our CPVT knockin mice. Strikingly, OGTTs were markedly abnormal in both CPVT patients and in 2 murine models of human CPVT (RyR2-R2474S and RyR2-N2368I). Moreover, glucose-stimulated insulin secretion evaluated ex vivo was reduced in isolated islets from CPVT mice with leaky RyR2 channels.

Mounting evidence indicates that ER and mitochondria are highly dynamic organelles that are structurally and functionally related (3). The activation of ER stress response has been demonstrated to cause mitochondrial dysfunction (38), triggering oxidative stress and further exacerbating ER stress (39). Our CPVT models displayed a mild ER stress response in pancreatic $\beta$ cells associated with chronic $\mathrm{ER} \mathrm{Ca}^{2+}$ depletion due to leaky RyR2 channels, with the upregulation of BiP and unspliced/spliced XBP1, but 
A

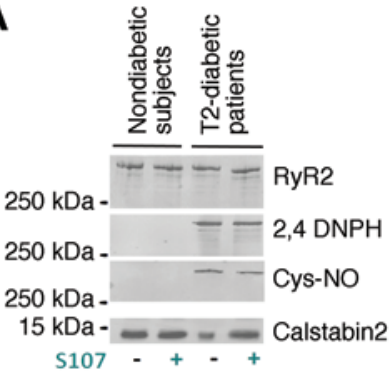

B
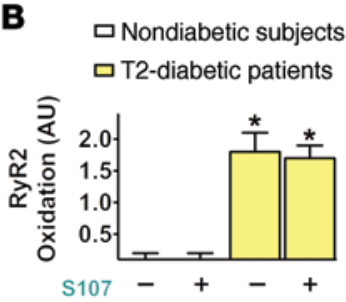

$\mathbf{F}$

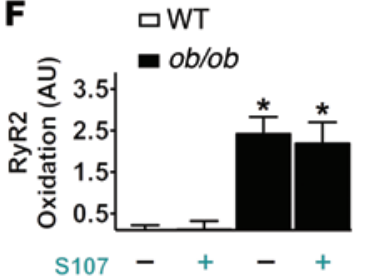

C

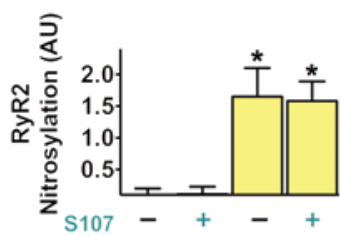

G

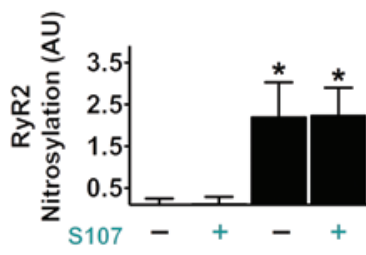

D

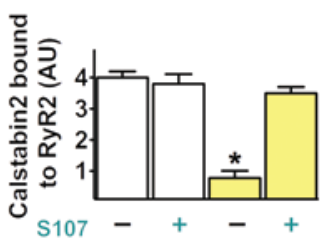

I

$$
\begin{aligned}
& \text { D } o b / o b+\text { vehicle } \\
& \text { 口 } o b / o b+S 107
\end{aligned}
$$

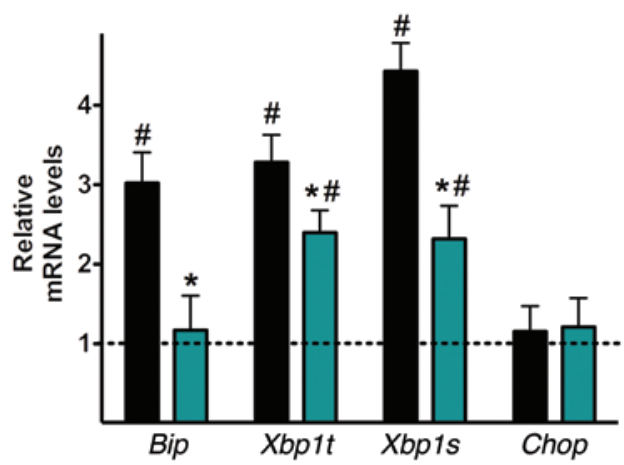

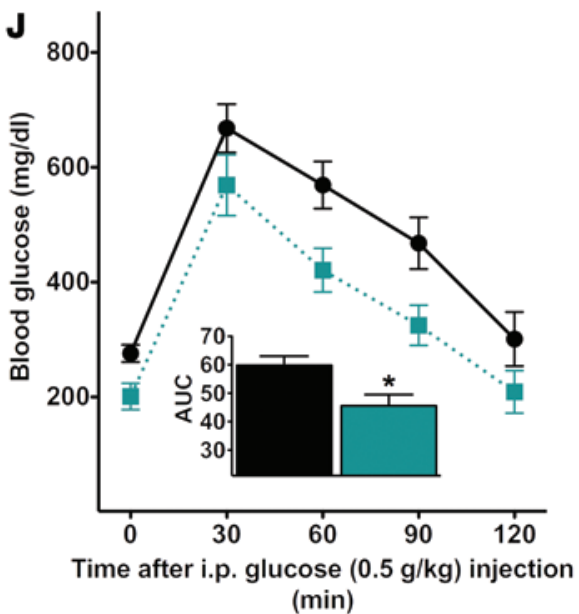

$\mathbf{K}$

H

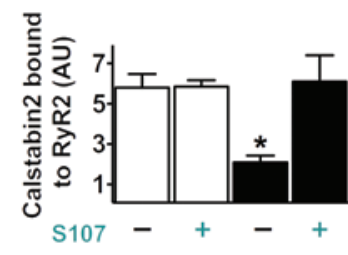

Figure 5. Fixing the RyR2-mediated ER Ca+ leak restores calstabin2 binding to RyR2 in human diabetic islets and improves ER stress response, glucose tolerance, and insulin secretion in diabetic mice. (A) Representative immunoblots of pancreatic islets from healthy subjects (nondiabetic) and patients with T2DM. Islets were treated with or without S107 (10 $\mu$ M for 4 hours). (B-D) Quantification (from triplicate experiments, donors' clinical information is given in Supplemental Table 4) of RyR2 oxidation (B), RyR2 nitrosylation (C), and levels of calstabin2 bound to RyR2 (D). ${ }^{*} P<0.05$ vs. nondiabetic, ANOVA. (E-H) Immunoblots of pancreatic islets from ob/ob mice treated with or without $\mathrm{S107}$ (50 mg/kg/d in drinking water for 4 weeks); representative immunoblots (E) and quantification (triplicate experiments) of RyR2 oxidation (F), RyR2 nitrosylation (G), and calstabin2 bound to RyR2 (H). ${ }^{*} P<0.05$ vs. untreated WT, ANOVA. (I) ER stress response in murine islets evaluated in terms of Bip, total and spliced Xbp1, and Chop by real-time RT-qPCR analysis of total RNA, relative to untreated WT mice (horizontal dashed line), using $\beta$ actin as internal standard. Primer sequences are reported in Supplemental Table 3. Each bar represents mean \pm SEM of 4 independent experiments, each performed in triplicate. ${ }^{*} P<0.05$ vs. ob/ob plus vehicle; ${ }^{\#} P<0.05$ vs. WT, 2-tailed Student's $t$ test. (J and K) Effects of chronic 5107 treatment ( 50 mg/kg/d in drinking water for 4 weeks) in ob/ob mice on glucose tolerance in vivo (J) and insulin release from isolated pancreatic islets (K). All data are shown as mean \pm SEM. $n \geq 6$ animals/group. ${ }^{*} P<0.05$ vs. ob/ob plus vehicle, 2-tailed Student's $t$ test.

with normal levels of CHOP, which is considered the key player in the activation of apoptotic pathways elicited by ER stress (40). We speculate that in the long term, mitochondrial dysfunction could have a detrimental effect on $\beta$ cell survival, which may eventually become apparent only in aged mice. Indeed, a recent study has demonstrated that $\mathrm{Ca}^{2+}$ depletion pharmacologically obtained by thapsigargin application is associated with activation of ER stress, causing upregulation of both BiP and CHOP in a dosedependent manner, eventually inducing cell death (41). Furthermore, our data show that, in addition to ER stress due to ER $\mathrm{Ca}^{2+}$ depletion, primarily triggered by leaky RyR2 channels, another factor contributing to impaired insulin secretion is the reduction in mitochondrial ATP production, attributable to decreased mitochondrial $\mathrm{Ca}^{2+}$ uptake secondary to $\mathrm{ER} \mathrm{Ca}^{2+}$ depletion. Indeed, mitochondrial $\mathrm{Ca}^{2+}$ depletion can reduce ATP production because the activity of several of the enzymes in the Krebs cycle is regulated by $\mathrm{Ca}^{2+}(17,42)$.

Mitochondrial metabolism orchestrates fuel-stimulated insulin release in pancreatic $\beta$ cells by finely tuning the rate of ATP generation $(4,32,33,35,38,43-45)$. Compared with WT, islets of Langerhans from mice with leaky RyR2 channels exhibited ultrastructural and functional abnormalities and decreased levels of ATP. Our findings are consistent with mitochondrial alterations described in $\beta$ cells from T2DM patients (46) and may also 


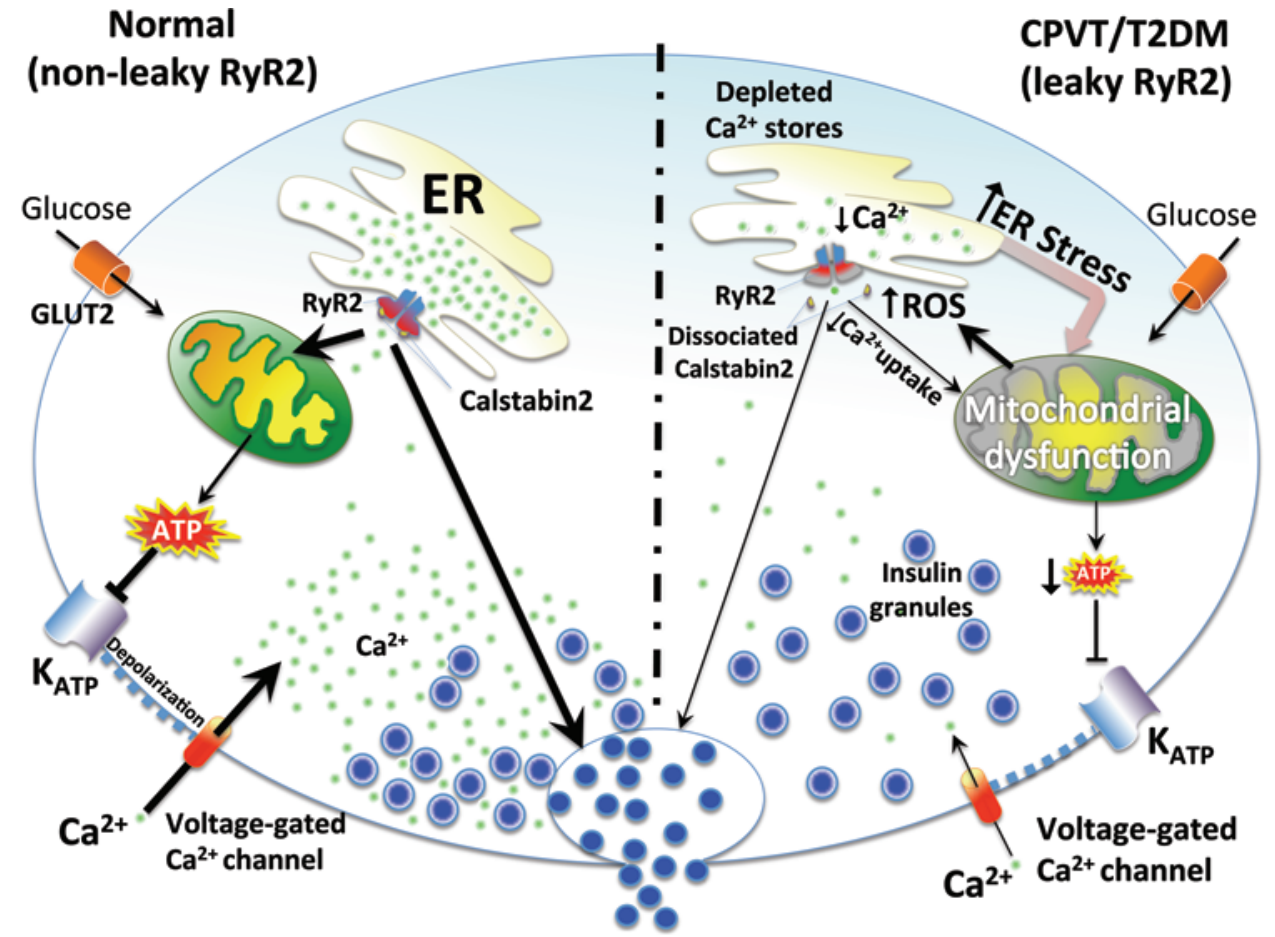

Figure 6. Model of RyR2-mediated ER $\mathrm{Ca}^{2+}$ leak in the pancreatic $\beta$ cell. Chronic intracellular $\mathrm{Ca}^{2+}$ leak via RyR2 channels causes store depletion, triggering ER stress and mitochondrial dysfunction, leading to reduced ATP synthesis and eventually decreased glucosestimulated insulin release, indicating an altered metabolism-secretion coupling. Impaired mitochondrial fitness also leads to increased production of ROS, which trigger redox modifications of RyR2 alongside calstabin2 dissociation, thereby exacerbating the $\mathrm{Ca}^{2+}$ leak. GLUT2, glucose transporter 2 . explain, at least in part, why RyR2 are oxidized in CPVT islets. Alterations in mitochondrial dynamics have been reported among the mechanisms underlying the development of T2DM and obesity $(38,43,45)$. Further supporting our results, RyR2 channels on the ER have been recently shown to be in close proximity to mitochondria in pancreatic $\beta$ cells $(47,48)$, suggesting that depletion of intracellular $\mathrm{Ca}^{2+}$ store via RyR2 leak could affect mitochondrial function. The abnormal upregulation of PGC-1 $\alpha$ observed in both CPVT and $o b / o b$ mice represents a shared feature of numerous models of T2DM and has been proposed as a pathophysiological link connecting mitochondrial dysfunction and $\beta$ cell failure (49). The reduced ATP production detected in CPVT mice in response to both glucose and pyruvate most likely contributes to glucose intolerance via decreased insulin secretion. Indeed, both CPVT mutations investigated in the present study caused an upregulation, which might also be compensatory, of $\mathrm{K}_{\text {ATP }}$ channel activity. We did not specifically evaluate other potential electrophysiological modifications in channel activity, which we speculate to be elevated in CPVT mice, consistent with the demonstration of gain-of-function mutations in the Kir6.2 gene in monogenic forms of diabetes $(5,50)$. Moreover, we found that pharmacological blockade of $\mathrm{K}_{\text {ATP }}$ channels with glyburide rescued insulin release. These results suggest a scenario in which the chronic $\mathrm{ER} \mathrm{Ca}^{2+}$ leak triggers ER stress and mitochondrial dysfunction, causing a bioenergetic deficit with decreased ATP synthesis (Figure 6).

T2DM represents a heterogeneous disease that results from a complex inheritance-environment interaction (51-55). GWAS have identified components of the genetic architecture of T2DM susceptibility (56-58). Notably, a GWAS focused on African Americans revealed an association between T2DM and a single nucleotide polymorphism located intergenically between RYR2 and 5-methyltetrahydrofolate-homocysteine (59). Previously, the association between RYR2 and T2DM was suggested in the first GWAS that identified risk loci for T2DM (56); however, RYR2 had been subsequently excluded (56).

One limitation of our study is that the genetically altered mice used are not pancreatic $\beta$ cell specific, but general knockins. On the one hand, this accurately reflects the human condition, as the CPVT patients are heterozygous for RYR2 mutations $(23,24)$, which are expressed throughout the body (17). However, we cannot exclude the possibility that leaky RyR2 in other organs also contribute to the altered glucose metabolism observed in humans and mice with RYR2 mutations. Nonetheless, the finding that the knockin of single amino acid missense mutations causes a phenotype in mice similar to that in humans (CPVT and glucose intolerance) provides strong genetic evidence supporting a central role for mutant leaky RyR2 in the pathogenesis of cardiac arrhythmias and altered glucose metabolism. Additionally, the restoration of insulin secretion and glucose homeostasis obtained with chronic S107 treatment provides further support for the RyR2-mediated leak as the culprit in both of these abnormalities. We did not perform an arginine test to assess the maximal insulin response (6062). Such a test could have helped distinguish between the role of RyR2 in acute function versus the maintenance of $\beta$ cell mass (63).

The $o b / o b$ mouse used in this study to test in vivo the effects of S107 on insulin secretion and glucose homeostasis is a well-established model of T2DM $(33,34,62,64)$. However, $\beta$ cell failure is not considered the main trigger of diabetes in this murine model, in which a major pathophysiological role is played by insulin resistance; therefore, modifications in RyR2 observed in pancreatic islets from these mice could represent an epiphenomenon of a prolonged hyperglycemic status. We did not investigate mitochondrial morphology in $\beta$ cells from obese mice, which has been reported to be profoundly altered (34), and we did not specifically test the potential beneficial effects of Rycals in this context or the possibility of systemic effects of $\mathrm{S} 107$ on peripheral tissues. 
The present study indicates a functional role for RyR2 in metabolism-secretion coupling both in mice and in humans. T2DM represents a potent risk factor for the development of cardiovascular disease (65-69). Since RyR2 channels are chronically leaky in heart failure $(17,20)$, our findings raise the possibility that the relationship between diabetes and cardiovascular disorders may be more complex than previously appreciated in that T2DM is both a risk factor for and a sequela of heart disease. Taken together, our data provide compelling evidence that intracellular $\mathrm{Ca}^{2+}$ leak via RyR2 channels induces glucose intolerance associated with pancreatic $\beta$ cell ER stress, mitochondrial dysfunction, and decreased insulin secretion.

\section{Methods}

Human studies. All recruited subjects were of mixed European descent and unrelated. Glucose metabolism was assessed by means of fasting blood glucose and standard OGTT $(75 \mathrm{~g}$ glucose in $200 \mathrm{ml}$ water). The number of participants was determined with an a priori power analysis using a standard for adequacy of $80 \%$ to reject the null hypothesis of zero correlation. Subjects with known disorders other than CPVT were not enrolled. Human islets used in this study were obtained from normal and diabetic (T2DM) cadaveric donors from the Islet Resource Facility, Transplantation Division, Southern Tissue Center, University of Alabama at Birmingham (Birmingham, Alabama, USA) and from Prodo Laboratories Inc. Characteristics of donors are reported in Supplemental Table 4. S107 treatment consisted of incubation of human islets with the drug $(10 \mu \mathrm{M})$ for 4 hours.

Animal experiments. The $o b / o b$ mice were purchased from Jackson Laboratories. RyR2-R2474S and RyR2-N2386I mice were generated as described previously (27). Animals were maintained in a specific pathogen-free animal facility on a 12-hour light/12-hour dark cycle at an ambient temperature of $21 \pm 2^{\circ} \mathrm{C}$. They were given free access to water and food. $\mathrm{S} 107$ was diluted in the drinking water. The optimal dosage was determined based on preliminary time-course and doseresponse assays. Age-matched male mice were used for all animal experiments, which were conducted by operators who were blinded to the genotypes of the mice. Glucose, insulin, C-peptide, and glucagon (Wako Diagnostics and Ultrasensitive ELISA, Mercodia) were determined as previously described (1). Plasma nonesterified fatty acids and triglycerides were assessed in fasted animals using commercially available kits (Wako Diagnostics and Sigma-Aldrich). For morphometric analysis of pancreatic islets, at least 6 animals (4 months old) per group were analyzed. The staining for insulin and glucagon was performed according to established protocols (1). For each pancreas, several sections spaced approximately $160 \mu \mathrm{m}$ apart were covered systematically and images were analyzed using Fiji (70).

Mouse pancreatic islet isolation. Mouse pancreatic islet isolation was performed as previously described (1). Briefly, islets of Langerhans were isolated by collagenase P digestion (Roche Applied Sciences) in a shaking water bath $\left(37^{\circ} \mathrm{C}\right)$ for 5 to 8 minutes. The digested pancreas was treated with DNase I (New England BioLabs). After Histopaque gradient purification, the islets were hand picked under a stereomicroscope and cultured at $37^{\circ} \mathrm{C}$ with $95 \%$ air and $5 \% \mathrm{CO}_{2}$ in RPMI 1640 (1). In some experiments, islets were treated with glucose (5.5 and $16.7 \mathrm{mM})$, glyburide $(10 \mu \mathrm{M})$, methyl pyruvate $(10 \mathrm{mM})$, or L-leucine $(10 \mathrm{mM})$ and glutamine $(2 \mathrm{mM})$, all from Sigma-Aldrich. Islet ATP content was determined using a quantitative bioluminescence assay (Sigma-Aldrich), as described $(71,72)$. ROS production was assessed using 5-(and-6) chloromethyl-2',7'-dichlorodihydrofluorescein diacetate $\left(\mathrm{CM}-\mathrm{H}_{2} \mathrm{DCFDA}\right.$; Thermo Fisher Scientific). Aconitase activity was evaluated using a commercially available assay (Cayman Chemical) following the manufacturer's instructions.

$\mathrm{Ca}^{2+}$ measurements. $\mathrm{Ca}^{2+}$ imaging assays were performed as previously described $(21,22,26,27,73)$. Briefly, islets were dispersed using $5 \mathrm{mg} / \mathrm{ml}$ dispase (Roche) attached on poly-L-ornithine-coated (PORNcoated) (Sigma-Aldrich) glass-bottom culture dishes (MatTek Corp.) loaded with Fura- $2 \mathrm{AM}\left(5 \mu \mathrm{M}, 15\right.$ minutes, $\left.37^{\circ} \mathrm{C}\right)$. Images were obtained using a DeltaScan dual excitation fluorescence imaging system (Photon Technology International) on the stage of a Nikon A1 inverted microscope (Nikon Fluor, $\times 40$ objective, numerical aperture 1.3). A field of cells was illuminated alternately at 340 and $380 \mathrm{~nm}$ and emitted fluorescence $(510 \mathrm{~nm})$ images collected on line using a Coolsnap k4 CCD video camera (Princeton Instrument) connected to a Photon Technology International image processor. This provided an indication of $\mathrm{Ca}^{2+}$ content that was independent of dye concentration in the tissue.

NADH autofluorescence was imaged in unloaded islets excited at a wavelength of $340 \mathrm{~nm}$ and recorded at $510 \mathrm{~nm}$. Changes in intracellular $\mathrm{Ca}^{2+}$ were reflected in the ratio of fluorescence emission acquired above $510 \mathrm{~nm}$ in response to excitation at $340 \mathrm{~nm}$ and $380 \mathrm{~nm}$.

Mitochondrial $\mathrm{Ca}^{2+}$ was assessed using a confocal microscope (Zeiss LSM 5 Live, $\times 63$ oil immersion lens) in $\beta$ cells loaded with rhod- 2 AM $\left(3 \mu \mathrm{M}, 30\right.$ minutes, $\left.37^{\circ} \mathrm{C}\right)$, followed by washout and 1 hour rest at room temperature for de-esterification. Due to its delocalized positive charge, this dye accumulates preferentially within the mitochondrial matrix, where it is hydrolyzed and trapped. Fluorescence was detected using a pass-band filter of 545 to $625 \mathrm{~nm}$ in response to excitation at $542 \mathrm{~nm}$.

To measure $\mathrm{Ca}^{2+}$ leak in microsomes, pancreatic islets were homogenized in $10 \mathrm{mM}$ Tris-maleate buffer with protease inhibitors. The homogenate was centrifuged for 20 minutes at $4,000 \mathrm{~g}$. The supernatant was subsequently centrifuged for 30 minutes at $45,000 \mathrm{~g}$. The resulting pellet was then resuspended in $50 \mathrm{ml}$ of Tris-maleate buffer ( $\mathrm{pH}$ 7.4) containing $0.3 \mathrm{M}$ sucrose and $0.9 \% \mathrm{NaCl}$. The $\mathrm{Ca}^{2+}$ loading and leak assay was performed using microsomes $(150 \mu \mathrm{g})$ from islet samples in $1 \mathrm{ml}$ of buffer: $120 \mathrm{mM} \mathrm{K}$-gluconate, $7 \mathrm{mM} \mathrm{NaCl}$;

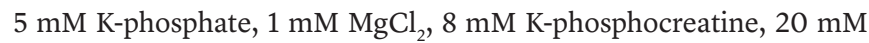
HEPES/K(OH), $\mathrm{pH} 7.2$, and 2 units/ml of creatine kinase and the $\mathrm{Ca}^{2+}$-sensitive fluorescent indicator Fluo-3 $(2.5 \mu \mathrm{M}) . \mathrm{Ca}^{2+}$ loading was initiated by adding $0.8 \mathrm{mM}$ ATP to activate the $\mathrm{Ca}^{2+}$ uptake pump, after which thapsigargin $(0.8 \mu \mathrm{M})$ was added to block the $\mathrm{Ca}^{2+}$ pump. Ryanodine $(20 \mu \mathrm{M})$ was used to prove that the RyR channel was the specific source of the leak. The time course of $\mathrm{Ca}^{2+}$ uptake was monitored spectrophotometrically as the emission of Fluo-3 after excitation at $485 \mathrm{~nm}$. Changes of fluorescence intensity were recorded by dedicated software (FeliX version 2; Photon Technology International).

Transmission electron microscopy. Each pancreas was fixed in $2.5 \%$ glutaraldehyde in $0.1 \mathrm{M}$ Sørensen's buffer and post-fixed in 1\% $\mathrm{OsO}_{4}$. Following dehydration, samples were embedded in Lx-112 (Ladd Research Industries). After cutting (ultramicrotome MT-7000), 60-nm sections were stained with uranyl acetate and lead citrate and visualized (JEM-1200 EXII, JEOL). From 8 to 10 randomly obtained sections for each pancreas were used for morphological analyses of mitochondria and secretory granules. $\beta$ Cell secretory granules are polymorphous and contain crystalline cores surrounded by a prominent electron-lucent halo beneath the limiting membrane and can be distinguished from $\alpha$ cell granules, which are typically relatively small- 
er and display a more electron-dense core with a very limited electrontransparent halo. To estimate mitochondrial size and cristae density, a computerized point grid was digitally layered over the micrographic images. All analyses were performed in a blinded fashion.

Immunoprecipitation and immunoblotting. Islet homogenates were lysed in $150 \mathrm{mM} \mathrm{NaCl}, 25 \mathrm{mM}$ Tris- $\mathrm{HCl}, \mathrm{pH}$ 7.5, 5 mM EDTA, 1\% NP-40, $0.4 \%$ deoxycholic acid, $1 \mathrm{mM} \mathrm{Na}_{3} \mathrm{VO}_{4}$, and complete protease inhibitors. RyR2 was immunoprecipitated from precleared lysates with $4 \mu \mathrm{g}$ anti-RyR2 antibody $(22,26,74)$. After 3 hours incubation, $30 \mu \mathrm{l}$ of $50 \%$ slurry of protein A-Sepharose were added to each sample and incubated for an additional hour at $4^{\circ} \mathrm{C}$. Precipitates were washed $4 \times$ with lysis buffer, size fractionated on SDS-PAGE, and immunoblotted. Oxidation and Cys-nitrosylation of RyR were determined as described (21). Immunoblotting was performed as previously described $(26,75$, 76) using the following antibodies: calstabin 2 (catalog sc-98742, Santa Cruz Biotechnology), Kir6.2 (catalog ab95317, Abcam), and GAPDH (catalog 2118, Cell Signaling Technology).

Real-time RT-qPCR. Real-time quantitative reverse-transcriptasePCR (RT-qPCR) was performed as described $(75,76)$. In brief, total RNA was isolated using TRIzol reagent (Thermo Fisher Scientific) in combination with the RNeasy Mini kit (QIAGEN) followed by DNase treatment, and cDNA was synthesized by means of a Thermo-Script RT-PCR System (Thermo Fisher Scientific), according to the manufacturer's instruction. After reverse transcription, real-time qPCR was performed in triplicate using the SYBR green real-time PCR Master Mix Kit and quantified by built-in SYBR Green Analysis (Thermo Fisher Scientific). To determine mtDNA and nuclear DNA (nDNA) copy number, qPCR was performed on $30 \mathrm{ng}$ of total islet DNA in each qPCR reaction using primer pairs for mitochondrial and genomic loci. Two different primer pairs were used to quantify and confirm relative mtDNA/nDNA ratio: $12 \mathrm{~S}$ and $16 \mathrm{~S}$ for mtDNA, H19 and $18 \mathrm{~S}$ for nDNA. Samples were measured in triplicate, and results were confirmed by at least 3 independent experiments. All primer sequences (SigmaAldrich) for gene analyses are listed in Supplemental Table 3.
Statistics. All results are presented as mean \pm SEM. Statistical analysis was performed using the unpaired Student's $t$ test, 2-tailed (for 2 groups), and 1-way ANOVA with Tukey-Kramer post hoc correction (for groups of 3 or more) unless otherwise indicated. $P<0.05$ was considered to be statistically significant.

Study approval. The IACUC of Columbia University Medical Center approved all animal study procedures, which were performed according to NIH guidelines. Human venous whole blood samples were obtained from volunteers who provided signed, informed consent, according to Declaration of Helsinki principles. All studies complied with the EU Protocol on Human Rights and Biomedicine.

\section{Acknowledgments}

The authors thank D. Accili, A.W. Ferrante Jr., and the members of their laboratories (Naomi Berrie Diabetes Center, Columbia University) for helpful discussions; M. Cacheux, D.D. Chang, B. Chen, H.M. Colecraft, B.W. Osborne, L. Xu, J. Yang, Q. Yuan, R. Zalk, and P. Zoppoli (Columbia University) for technical assistance; and G. Condorelli (Humanitas Clinical and Research Center, Rozzano, Italy) and G. Iaccarino (Salerno University, Salerno, Italy) for insightful comments. This work was supported by the American Heart Association (AHA) (13POST16810041 to G. Santulli), the Schaefer Foundation, the Phillip Foundation (to A. Lacampagne, currently on sabbatical in the Marks laboratory), and the NIH (R01HL061503, R01HL102040, R01AR060037 to A.R. Marks).

Address correspondence to: Gaetano Santulli or Andrew R. Marks, Columbia University Medical Center, Department of Physiology and Cellular Biophysics, College of Physicians and Surgeons, Clyde and Helen Wu Center for Molecular Cardiology, 1150 Saint Nicholas Avenue, R513, New York, New York 10032, USA. Phone: 212.851.5348; E-mail: gs2620@cumc.columbia.edu or gsantulli001@gmail.com (G. Santulli). Phone: 212.851.5340; E-mail:arm42@columbia.edu (A.R. Marks).
1. Santulli G, et al. Age-related impairment in insulin release: the essential role of $\beta(2)$-adrenergic receptor. Diabetes. 2012;61(3):692-701.

2. Prentki M, Nolan CJ. Islet $\beta$ cell failure in type 2 diabetes. J Clin Invest. 2006;116(7):1802-1812.

3. Rutter GA, Pinton P. Mitochondria-associated endoplasmic reticulum membranes in insulin signaling. Diabetes. 2014;63(10):3163-3165.

4. Wollheim CB, Maechler P. $\beta$-Cell mitochondria and insulin secretion: messenger role of nucleotides and metabolites. Diabetes. 2002;51(suppl 1):S37-S42.

5. Ashcroft FM, Rorsman P. K(ATP) channels and islet hormone secretion: new insights and controversies. Nat Rev Endocrinol. 2013;9(11):660-669.

6. De Marchi U, Thevenet J, Hermant A, Dioum E, Wiederkehr A. Calcium co-regulates oxidative metabolism and ATP synthase-dependent respiration in pancreatic $\beta$ cells. J Biol Chem. 2014;289(13):9182-9194.

7. Dadi PK, Vierra NC, Ustione A, Piston DW, Colbran RJ, Jacobson DA. Inhibition of pancreatic $\beta$-cell $\mathrm{Ca}^{2+} /$ calmodulin-dependent protein kinase II reduces glucose-stimulated calcium influx and insulin secretion, impairing glucose tolerance. J Biol Chem. 2014;289(18):12435-12445.
8. Nolan CJ, Madiraju MS, Delghingaro-Augusto V, Peyot ML, Prentki M. Fatty acid signaling in the $\beta$-cell and insulin secretion. Diabetes. 2006;55(suppl 2):S16-S23.

9. Henquin JC, Nenquin M, Stiernet P, Ahren B. In vivo and in vitro glucose-induced biphasic insulin secretion in the mouse: pattern and role of cytoplasmic $\mathrm{Ca}^{2+}$ and amplification signals in $\beta$-cells. Diabetes. 2006;55(2):441-451.

10. Namkung Y, et al. Requirement for the L-type $\mathrm{Ca}(2+)$ channel alpha(1D) subunit in postnatal pancreatic $\beta$ cell generation. J Clin Invest. 2001;108(7):1015-1022.

11. Gilon P, Chae HY, Rutter GA, Ravier MA. Calcium signaling in pancreatic $\beta$-cells in health in Type 2 diabetes. Cell Calcium. 2014;56(5):340-361.

12. Henquin JC, Mourad NI, Nenquin M. Disruption and stabilization of $\beta$-cell actin microfilaments differently influence insulin secretion triggered by intracellular $\mathrm{Ca}^{2+}$ mobilization or store-operated $\mathrm{Ca}^{2+}$ entry. FEBS Lett. 2012;586(1):89-95.

13. Zhang Q, et al. R-type $\mathrm{Ca}(2+)$-channel-evoked CICR regulates glucose-induced somatostatin secretion. Nat Cell Biol. 2007;9(4):453-460.

14. Islam MS. Calcium signaling in the islets. $A d v \operatorname{Exp}$
Med Biol. 2010;654:235-259.

15. Bruton JD, et al. Ryanodine receptors of pancreatic $\beta$-cells mediate a distinct contextdependent signal for insulin secretion. FASEB J. 2003;17(2):301-303.

16. Johnson JD, Kuang S, Misler S, Polonsky KS. Ryanodine receptors in human pancreatic $\beta$ cells: localization and effects on insulin secretion. FASEB J. 2004;18(7):878-880.

17. Santulli G, Marks AR. Essential roles of intracellular calcium release channels in muscle, brain, metabolism, aging. Curr Mol Pharmacol. In press.

18. Zalk R, et al. Structure of a mammalian ryanodine receptor. Nature. 2015;517(7532):44-49.

19. Brillantes AB, et al. Stabilization of calcium release channel (ryanodine receptor) function by FK506-binding protein. Cell. 1994;77(4):513-523.

20. Marks AR. Calcium cycling proteins and heart failure: mechanisms and therapeutics. JClin Invest. 2013;123(1):46-52.

21. Umanskaya A, Santulli G, Xie W, Andersson DC, Reiken SR, Marks AR. Genetically enhancing mitochondrial antioxidant activity improves muscle function in aging. Proc Natl Acad Sci US A. 2014;111(42):15250-15255. 
22. Fauconnier J, et al. Leaky RyR2 trigger ventricular arrhythmias in Duchenne muscular dystrophy. Proc Natl Acad Sci U S A. 2010;107(4):1559-1564.

23. Lehnart SE, et al. Sudden death in familial polymorphic ventricular tachycardia associated with calcium release channel (ryanodine receptor) leak. Circulation. 2004;109(25):3208-3214.

24. Leenhardt A, Denjoy I, Guicheney P. Catecholaminergic polymorphic ventricular tachycardia. Circ Arrhythm Electrophysiol. 2012;5(5):1044-1052.

25. Santulli G. Classification of syncope-producing cardiac arrhythmias. In: Silva E, Cruz, G, eds. Comas and Syncope: Causes, Prevention and Treatment. New York, New York, USA: Nova Science Publisher; 2012:167-177.

26. Yuan Q, et al. Functional role of Calstabin2 in agerelated cardiac alterations. Sci Rep. 2014;(4):7425.

27. Xie W, Santulli G, Guo X, Gao M, Chen BX, Marks AR. Imaging atrial arrhythmic intracellular calcium in intact heart. J Mol Cell Cardiol. 2013;64:120-123.

28. Bellinger AM, et al. Remodeling of ryanodine receptor complex causes "leaky" channels: a molecular mechanism for decreased exercise capacity. Proc Natl Acad Sci U S A. 2008;105(6):2198-2202.

29. Noguchi N, et al. FKBP12.6 disruption impairs glucose-induced insulin secretion. Biochem Biophys Res Commun. 2008;371(4):735-740.

30. Cardozo AK, et al. Cytokines downregulate the sarcoendoplasmic reticulum pump $\mathrm{Ca}^{2+}$ ATPase $2 \mathrm{~b}$ and deplete endoplasmic reticulum $\mathrm{Ca}^{2+}$, leading to induction of endoplasmic reticulum stress in pancreatic $\beta$-cells. Diabetes. 2005;54(2):452-461.

31. O'Neill CM, et al. Circulating levels of IL$1 \mathrm{~B}+\mathrm{IL}-6$ cause ER stress and dysfunction in islets from prediabetic male mice. Endocrinology. 2013;154(9):3077-3088.

32. Vetterli L, et al. Delineation of glutamate pathways and secretory responses in pancreatic islets with $\beta$-cell-specific abrogation of the glutamate dehydrogenase. Mol Biol Cell. 2012;23(19):3851-3862.

33. Zhang CY, et al. Uncoupling protein-2 negatively regulates insulin secretion and is a major link between obesity, $\beta$ cell dysfunction, and type 2 diabetes. Cell. 2001;105(6):745-755.

34. Yoshihara E, et al. Disruption of TBP-2 ameliorates insulin sensitivity and secretion without affecting obesity. Nat Commun. 2010;1:127.

35 . Krauss S, et al. Superoxide-mediated activation of uncoupling protein 2 causes pancreatic $\beta$ cell dysfunction. J Clin Invest. 2003;112(12):1831-1842.

36. Gier B, et al. Suppression of KATP channel activity protects murine pancreatic $\beta$ cells against oxidative stress. JClin Invest. 2009;119(11):3246-3256.

37. Ye Y, et al. Designing calcium release channel inhibitors with enhanced electron donor properties: stabilizing the closed state of ryanodine receptor type 1. Mol Pharmacol. 2012;81(1):53-62.

38. Mitchell T, et al. Dysfunctional mitochondrial bioenergetics and oxidative stress in Akita(+/ Ins2)-derived $\beta$-cells. Am J Physiol Endocrinol Metab. 2013;305(5):E585-E599.

39. Tang C, et al. Glucose-induced $\beta$ cell dysfunction in vivo in rats: link between oxidative stress and endoplasmic reticulum stress. Diabetologia 2012;55(5):1366-1379.

40. Lombardi A, Inabnet WB 3rd, Owen R, Faren- holtz KE, Tomer Y. Endoplasmic reticulum stress as a novel mechanism in amiodarone-induced destructive thyroiditis. JClin Endocrinol Metab 2015;100(1):E1-E10.

41. Hara T, Mahadevan J, Kanekura K, Hara M, Lu S, Urano F. Calcium efflux from the endoplasmic reticulum leads to $\beta$-cell death. Endocrinology. 2014;155(3):758-768.

42. Perocchi F, et al. MICU1 encodes a mitochondrial $\mathrm{EF}$ hand protein required for $\mathrm{Ca}(2+)$ uptake. Nature. 2010;467(7313):291-296.

43. MacDonald PE, Joseph JW, Rorsman P. Glucose-sensing mechanisms in pancreatic $\beta$-cells. Philos Trans R Soc Lond B Biol Sci. 2005;360(1464):2211-2225.

44. Wiederkehr A, Wollheim CB. Mitochondrial signals drive insulin secretion in the pancreatic $\beta$-cell. Mol Cell Endocrinol. 2012;353(1-2):128-137.

45. Jitrapakdee $S$, Wutthisathapornchai A, Wallace JC, MacDonald MJ. Regulation of insulin secretion: role of mitochondrial signalling. Diabetologia. 2010;53(6):1019-1032.

46. Anello M, et al. Functional and morphological alterations of mitochondria in pancreatic $\beta$ cells from type 2 diabetic patients. Diabetologia. 2005;48(2):282-289.

47. Dror V, et al. Glucose and endoplasmic reticulum calcium channels regulate HIF- $1 \beta$ via presenilin in pancreatic $\beta$-cells. J Biol Chem. 2008;283(15):9909-9916.

48. Johnson JD, Bround MJ, White SA, Luciani DS. Nanospaces between endoplasmic reticulum and mitochondria as control centres of pancreatic $\beta$-cell metabolism and survival. Protoplasma. 2012;249(suppl 1):S49-S58.

49. Yoon JC, et al. Suppression of beta cell energy metabolism and insulin release by PGC- $1 \alpha$. Dev Cell. 2003;5(1):73-83.

50. Shimomura K, et al. Mutations at the same residue (R50) of Kir6.2 (KCNJ11) that cause neonatal diabetes produce different functional effects. Diabetes. 2006;55(6):1705-1712.

51. Bonnefond A, et al. Rare MTNR1B variants impairing melatonin receptor $1 \mathrm{~B}$ function contribute to type 2 diabetes. Nat Genet. 2012;44(3):297-301.

52. Hu FB, et al. Diet, lifestyle, and the risk of type 2 diabetes mellitus in women. $N$ Engl J Med. 2001;345(11):790-797.

53. Langenberg C, et al. Gene-lifestyle interaction and type 2 diabetes: the EPIC interact casecohort study. PLoS Med. 2014;11(5):e1001647.

54. Saxena R, et al. Genetic variation in GIPR influences the glucose and insulin responses to an oral glucose challenge. Nat Genet. 2010;42(2):142-148.

55. Fadista J, et al. Global genomic and transcriptomic analysis of human pancreatic islets reveals novel genes influencing glucose metabolism. Proc Natl Acad Sci U S A . 2014;111(38):13924-13929.

56. Sladek R, et al. A genome-wide association study identifies novel risk loci for type 2 diabetes. Nature. 2007;445(7130):881-885.

57. Vaxillaire M, et al. Type 2 diabetes-related genetic risk scores associated with variations in fasting plasma glucose and development of impaired glucose homeostasis in the prospective DESIR study. Diabetologia. 2014;57(8):1601-1610.

58. DIAbetes Genetics Replication Meta-Analysis
(DIAGRAM) Consortium, et al. Genome-wide trans-ancestry meta-analysis provides insight into the genetic architecture of type 2 diabetes susceptibility. Nat Genet. 2014;46(3):234-244.

59. Palmer ND, et al. A genome-wide association search for type 2 diabetes genes in African Americans. PLoS One. 2012;7(1):e29202.

60. Pontoglio M, et al. Defective insulin secretion in hepatocyte nuclear factor $1 \alpha$-deficient mice. JClin Invest. 1998;101(10):2215-2222.

61. Kulkarni RN, et al. Altered function of insulin receptor substrate-1-deficient mouse islets and cultured $\beta$-cell lines. JClin Invest. 1999;104(12):R69-R75.

62. Wu Y, et al. Growth hormone receptor regulates $\beta$ cell hyperplasia and glucose-stimulated insulin secretion in obese mice. J Clin Invest. 2011;121(6):2422-2426

63. Robertson RP, et al. Assessment of $\beta$-cell mass alpha- beta-cell survival function by arginine stimulation in human autologous islet recipients. Diabetes. 2014;64(2):565-572.

64. Kerouz NJ, Horsch D, Pons S, Kahn CR. Differential regulation of insulin receptor substrates-1 and -2 (IRS-1 and IRS-2) and phosphatidylinositol 3-kinase isoforms in liver and muscle of the obese diabetic (ob/ob) mouse. JClin Invest. 1997;100(12):3164-3172

65. Santulli G. Coronary heart disease risk factors and mortality. JAMA. 2012;307(11):1137.

66. Sardu C, Marfella R, Santulli G. Impact of diabetes mellitus on the clinical response to cardiac resynchronization therapy in elderly people. JCardiovasc Transl Res. 2014;7(3):362-368.

67. Santulli G. Thrombolysis outcomes in acute ischemic stroke patients with prior stroke and diabetes mellitus. Neurology. 2012;78(11):840.

68. Badimon L, Hernandez Vera R, Vilahur G. Determinants of cardiovascular risk in diabetes beyond hyperglycemia. J Cardiovasc Dis. 2013;1(2):53-62.

69. Santulli G. Beta-blockers in diabetic patients with heart failure. JAMA Intern Med. In press.

70. Santulli G, et al. CaMK4 gene deletion induces hypertension. JAm Heart Assoc. 2012;1(4):e001081.

71. Dukes ID, et al. Defective pancreatic $\beta$-cell glycolytic signaling in hepatocyte nuclear factor-1 $\alpha$-deficient mice. J Biol Chem . 1998;273(38):24457-24464.

72. Fusco A, et al. Mitochondrial localization unveil a novel role for GRK2 in organelle biogenesis. Cell Sig. 2012;24(2):468-475.

73. Johnson JD, et al. Increased islet apoptosis in $\mathrm{Pdx}^{+/-}$mice. J Clin Invest. 2003;111(8):1147-1160.

74. Cheung JW, et al. Short-coupled polymorphic ventricular tachycardia at rest linked to a novel ryanodine receptor (RyR2) mutation: leaky RyR2 channels under non-stress conditions. Int J Cardiol. 2015;180:228-236.

75. Sorriento D, Santulli G, Fusco A, Anastasio A, Trimarco B, Iaccarino G. Intracardiac injection of AdGRK5-NT reduces left ventricular hypertrophy by inhibiting NF- $\mathrm{KB}$-dependent hypertrophic gene expression. Hypertension. 2010;56(4):696-704.

76. Santulli G, et al. A selective microRNA-based strategy inhibits restenosis while preserving endothelial function. J Clin Invest. 2014;124(9):4102-4114. 